\title{
A rational framework for evaluating the next generation of vaccines against Mycobacterium avium subspecies paratuberculosis
}

\author{
John P. Bannantine ${ }^{1 *}$, Murray E. Hines $I^{2}$, Luiz E. Bermudez ${ }^{3}$, Adel M. Talaat ${ }^{4,5}$, Srinand Sreevatsan ${ }^{6}$, \\ Judith R. Stabel' ${ }^{1}$, Yung-Fu Chang ${ }^{7}$, Paul M. Coussens ${ }^{8}$, Raúl G. Barletta ${ }^{9}$, William C. Davis ${ }^{10}$, \\ Desmond M. Collins ${ }^{11}$, Yrjö T. Gröhn ${ }^{7}$ and Vivek Kapur ${ }^{12 *}$
}

1 Infectious Bacterial Diseases USDA-ARS, National Animal Disease Center, Ames, IA, USA

2 Tifton Veterinary Diagnostic and Investigational Lab, The University of Georgia, Tifton, GA, USA

${ }^{3}$ Departments of Microbiology and Biomedical Sciences, Oregon State University, Corvalis, OR, USA

${ }^{4}$ Department of Pathobiological Sciences, University of Wisconsin-Madison, Madison, WI, USA

${ }^{5}$ Department of Food Hygenie, Cairo University, Cairo, Egypt

${ }^{6}$ Veterinary Population Medicine Department, University of Minnesota, Minneapolis, MN, USA

7 Department of Population Medicine and Diagnostic Sciences, College of Veterinary Medicine, Cornell University, Ithaca, NY, USA

${ }^{8}$ Department of Animal Science, Michigan State University, Lansing, MI, USA

${ }^{9}$ School of Veterinary Medicine and Biomedical Sciences, University of Nebraska, Lincoln, NE, USA

${ }^{10}$ Department of Veterinary Microbiology, Washington State University, Pullman, WA, USA

$"$ AgResearch, Wallaceville, New Zealand

12 Department of Veterinary and Biomedical Sciences, Pennsylvania State University, University Park, PA, USA

\section{Edited by:}

Alfredo G. Torres, University of

Texas Medical Branch, USA

\section{Reviewed by:}

John T. Belisle, Colorado State

University, USA

Shen-An Hwang, University of Texas

Medical School, USA

*Correspondence:

John P. Bannantine,

USDA-Agricultural Research Service,

National Animal Disease Center,

1920 Dayton Ave., Ames, IA

50010, USA

e-mail: john.bannantine@

ars.usda.gov;

Vivek Kapur, Pennsylvania State

University, 205 Wartik Laboratory,

University Park, PA 16802, USA

e-mail:vkapur@psu.edu
Since the early 1980s, several investigations have focused on developing a vaccine against Mycobacterium avium subspecies paratuberculosis (MAP), the causative agent of Johne's disease in cattle and sheep. These studies used whole-cell inactivated vaccines that have proven useful in limiting disease progression, but have not prevented infection. In contrast, modified live vaccines that invoke a Th1 type immune response, may improve protection against infection. Spurred by recent advances in the ability to create defined knockouts in MAP, several independent laboratories have developed modified live vaccine candidates by transpositional mutation of virulence and metabolic genes in MAP. In order to accelerate the process of identification and comparative evaluation of the most promising modified live MAP vaccine candidates, members of a multi-institutional USDA-funded research consortium, the Johne's disease integrated program (JDIP), met to establish a standardized testing platform using agreed upon protocols. A total of 22 candidates vaccine strains developed in five independent laboratories in the United States and New Zealand voluntarily entered into a double blind stage gated trial pipeline. In Phase $\mathrm{I}$, the survival characteristics of each candidate were determined in bovine macrophages. Attenuated strains moved to Phase II, where tissue colonization of C57/BL6 mice were evaluated in a challenge model. In Phase III, five promising candidates from Phase I and II were evaluated for their ability to reduce fecal shedding, tissue colonization and pathology in a baby goat challenge model. Formation of a multi-institutional consortium for vaccine strain evaluation has revealed insights for the implementation of vaccine trials for Johne's disease and other animal pathogens. We conclude by suggesting the best way forward based on this 3-phase trial experience and challenge the rationale for use of a macrophage-to-mouse-to native host pipeline for MAP vaccine development.

Keywords: Johne's disease, Mycobacterium, vaccines, attenuated, transposons, animal models, genomics

\section{INTRODUCTION}

Johne's disease is caused by Mycobacterium avium subspecies paratuberculosis (hereafter referred to as MAP), an acid-fast bacillus that can be distinguished from other closely related mycobacteria by its unique requirement for the mycobactin $\mathrm{J}$ siderophore in artificial culture media (Merkal and Curran, 1974). This major veterinary pathogen can infect many species of animals
(Whittington et al., 2011), but its impact is felt most profoundly on commercial ruminant livestock such as cattle, sheep, goats and deer. In the United States (US) alone, current economic loses to the dairy industry are unknown, but previously was estimated at between 200 million and 1.5 billion dollars annually (Stabel, 1998). Almost three decades ago, the prevalence of Johne's disease in US dairy cattle was estimated at between 3 and $18 \%$ based 
on slaughterhouse surveys (Chiodini and Van Kruiningen, 1986; Merkal et al., 1987). A more recent national level serological survey conducted by the National Animal Health Monitoring System (NAHMS) in 2007 suggested MAP prevalence on US dairy farms had risen above $30 \%$. Unfortunately, this percentage continues to climb with the passage of time and implementation of more sensitive diagnostic tests. The most recent US dairy herd prevalence estimates are as high as 90\% (Lombard et al., 2013) and New Zealand farmed deer herd estimates are at 59\% (Stringer et al., 2013a). Collectively, these data suggest that MAP infection has long been endemic in the US and most likely across the world wherever dairy cows, sheep, goats and deer are intensively raised.

Vaccination against MAP infection has long been thought to be the best intervention strategy for this chronic and debilitating disease that is difficult to diagnose and slow to manifest. Animals actively shed large quantities of MAP before being diagnosed or exhibiting clinical signs-resulting in a transmission cycle that is very difficult to interrupt using traditional management strategies alone. Sub clinically infected animals transmit disease while appearing healthy and remaining undetectable by culture or PCR based approaches since these animals often shed MAP in a sporadic or intermittent manner. In stark contrast to the subversive "trickle and stealth" shedding pattern of MAP frequently observed in subclinical animals, there exist symptomatic, high shedder animals that excrete prodigious levels of organisms (up to $10^{8} \mathrm{CFU}$ per gram) in their feces, which provide the source of significant on-farm contamination (Pradhan et al., 2011). Diagnostic tests for Johne's disease are improving, but accurate detection of all infected animals, especially those that are early in infection and transmitting the organism within a herd, is still not possible. This fact makes test and cull strategies ineffective, except when targeting only high-shedding animals (Lu et al., 2008; Bastida and Juste, 2011). Therefore, it is widely recognized that unless animals can be detected early during infection, vaccination remains the best hope for controlling and preventing Johne's disease.

The ideal vaccine would completely prevent infection and/or promote protective immunity thus blocking both horizontal and vertical transmission. The current vaccines for Johne's disease fall far short of this high standard. MAP vaccines have been shown to be effective at lowering fecal shedding levels (Kalis et al., 2001; Faisal et al., 2013a), tissue colonization (Sweeney et al., 2009) or clinical disease incidence (Stringer et al., 2013b), but do not completely eliminate all three. Subunit vaccines against MAP are likely to obviate some of the shortcomings of whole-cell vaccines, such as severe inflammation and granuloma formation at the injection site. However, subunit vaccines that have been tested thus far have yielded incomplete protection results in murine models of infection (Koets et al., 2006; Stabel et al., 2012) and even when combinations of proteins are used in calves and goats (Koets et al., 2006; Kathaperumal et al., 2008, 2009). For example, MAP was colonized in the lymph node and spleen at similar levels in control and vaccinate mice using a protein cocktail (Stabel et al., 2012). Similarly, there were no significant MAP burden differences in the liver and mesenteric lymph node at 8 weeks post-challenge when immunizing mice with a $74 \mathrm{kDa}$ polyprotein, but there was a significant difference in the liver (Chen et al., 2008). However, additional studies using more animals, longer term to monitor protection (1-3 years) and field trials are needed. Unfortunately subunit vaccines are expensive to produce whereas the manufacture of attenuated mutants is low in cost and easy to produce. These considerations are even more important for food animal health. Thus, there are several considerations, but research in this area demonstrates the slow yet steady progress in vaccine development for Johne's disease.

Efforts to improve upon current vaccine formulations have long been hampered by the lack of standardized challenge models and the high costs associated with trials conducted in natural ovine, caprine, deer or bovine hosts. Progress is however being made, and standardized animal models (mouse, goat, cow) for Johne's disease have been developed by a group of international investigators under the auspices of the Johne's Disease Integrated Program (JDIP), a USDA-funded research consortium (Hines et al., 2007) to help facilitate comparisons between different vaccine trials. However, the costs and logistical challenges of MAP vaccine trials, particularly in the natural hosts, remain a major concern. And even with an efficacious vaccine, it is remotely possible that MAP could be maintained within a herd due to vertical transmission as noted in a recent vaccine modeling study ( $\mathrm{Lu}$ et al., 2013). Finally, there are negative implications of JD vaccination on both Johne's disease and bovine TB diagnostics as will be discussed further below.

Consistent with what is known about other intracellular pathogens, a MAP vaccine that drives the immune response to a proinflammatory Th1 profile and prevents a shift to the humoral Th2 response, maybe more effective in delaying progression of the disease to a clinical state (Coussens, 2004; Stabel, 2010). However, it has recently been suggested that humoral immunity may also be important against M. tuberculosis infection (Achkar and Casadevall, 2013). Nonetheless, current dogma suggests a late humoral response is not a protective immune response against Johne's disease. The focus of the JDIP vaccine studies was on live attenuated mutants rather than subunit or DNA vaccines because they are more likely to generate the beneficial Th1 immune response, which is considered the most protective against mycobacterial infections (Stabel, 2000; Hostetter et al., 2002). The cause for the switch from Th1 to Th2 immune responses in Johne's disease is unknown, however mathematical modeling of the bovine immune response to MAP suggests it may be attributed to extracellular MAP that persists outside of macrophages (Magombedze et al., 2014). Although these inferences need to be carefully tested experimentally, it is possible a mutant strain deficient in this capability might be the critical factor to an efficacious vaccine for Johne's disease.

Despite significant recent advances in the ability to make defined mutants, there have been only a limited number of trials testing live attenuated vaccines against MAP. These have been largely limited to in vitro studies or trials in the mouse (Scandurra et al., 2009; Settles et al., 2014), and except in a few cases (Scandurra et al., 2010; Faisal et al., 2013b), reflect a striking absence of robust and cost effective strategies for evaluating vaccine candidates in the relevant natural ruminant host. To help address this unmet need, and establish a rational framework based for the evaluation of novel vaccine candidates against MAP, 
we adopted an inclusive and collaborative approach that may also serve as a guide for future vaccine evaluation trials for MAP and other major animal pathogens. We here present the state-of-thefield summary for vaccination against Johne's disease, describe current candidate live attenuated mutants of MAP, and present an overview of the JDIP three-phase vaccine trial and lessons learned.

\section{MAP VACCINES: WHAT IS AVAILABLE?}

It is paradoxical that vaccination against MAP has not been widely used in the US given the high prevalence of Johne's disease in this country. Currently, there are at least three commercial Johne's disease vaccines worldwide, but only one (Mycopar $\left.{ }^{\circledR}\right)$ approved vaccine for use in the US (Bastida and Juste, 2011). Silirum ${ }^{\circledR}$ is produced by Zoetis and was used as the control vaccine in the JDIP mouse and goat trials discussed below. Mycopar ${ }^{\circledR}$ is manufactured by Boehringer Ingelheim Vetmedica Inc. whereas Gudair $^{\circledR}$ is produced by CZ Veterinaria in Spain and is being used in countries with large sheep populations (Bastida and Juste, 2011). Importantly, all three consist of an inactivated whole-cell preparation of either $M$. avium subspecies avium or subspecies paratuberculosis. As summarized by Bull et al. live attenuated strains were used in the 1960s and 1970s and then concerns about their use in the 1980s forced a shift to using killed vaccine formulations (Bull et al., 2013). Live vaccine strains can now be revisited with the technology used to create defined mutations.

What does the scientific literature report about the efficacy of these commercial MAP vaccines? In three dairy herd operations with Johne's disease, investigators showed that fewer Mycopar ${ }^{\circledR}$ vaccinated cattle had detectable fecal culture positive results compared with controls (Knust et al., 2013). This same vaccine also showed partial protection in a goat challenge model (Faisal et al., 2013a). However, the inactivated strain used in the Mycopar ${ }^{\circledR}$ formulation is not MAP, but an isolate called strain 18 that is nonetheless a member of the M. avium species. The manufacturer data sheet still lists Mycopar ${ }^{\circledR}$ as an "inactivated M. paratuberculosis bacterium suspended in oil." Interestingly, this parallelism occurs with the suboptimal response of BCG, derived from the related species $M$. bovis, as a vaccine against tuberculosis (Mangtani et al., 2014). Gudair consists of heat-killed MAP 316F and is designed for use in sheep and goats according to the manufacturer. Gudair ${ }^{\circledR}$ has been shown to be an effective vaccine in Merino sheep (Reddacliff et al., 2006). Vaccination not only delayed the onset of fecal shedding, but reduced mortality attributed to Johne's disease by $90 \%$ in the 200 vaccinated sheep. Silirum ${ }^{\circledR}$ is a killed strain of MAP, also related to $316 \mathrm{~F}$, that has been tested in Australian cattle. This vaccine has recently been shown to reduce prevalence of clinical disease, as measured by lymph node pathology and fecal culture at slaughter, in farmed deer in New Zealand (Stringer et al., 2013b). Currently, there are no subunit, live or DNA vaccines commercially available against Johne's disease.

\section{CONSTRUCTION OF ATTENUATED MAP MUTANTS}

Although attenuated MAP, generated by repeated subculture of the strain, had been used as a live vaccine long ago (Saxegaard and Fodstad, 1985), no defined mutants of MAP had been tested as a vaccine simply because no method was available for their construction. In 1995, the first concerted effort to develop the genetics of MAP was performed in Raúl Barletta's laboratory at the University of Nebraska (Foley-Thomas et al., 1995). Studies in his laboratory demonstrated for the first time that MAP could be transformed with foreign DNA as well as transfected with bacteriophage DNA. Furthermore, they found that MAP could be productively infected with the mycobacteriophage TM4 and later demonstrated the use of a thermosensitive derivative of TM4 to generate the first transposon mutant library (Harris et al., 1999). This methodological advance was adapted by other laboratories to create their own transposon mutant banks (Cavaignac et al., 2000; Shin et al., 2006). Phage-mediated techniques are now being applied to construct directed knockout mutations in MAP using allelic exchange. This technique worked well in $M$. tuberculosis, but the allelic exchange efficiency was low in MAP until investigators at Washington State University tweaked the protocol to increase efficiencies to generate $\Delta r e l A, \Delta l s r 2$, and $\Delta p k n G$ knockout strains (Park et al., 2008a). Now robust enough to be performed in other laboratories, this method enabled a more strategic approach to obtaining the precise knockout of a specific virulence gene rather than depend on random transposon mutagenesis and screening approaches to find a suitable knockout to try as a vaccine. The ppiA (Scandurra et al., 2010), relA, lsr2, pknG (Park et al., 2008a), leuD, mpt64, and secA2 genes (Chen et al., 2012) have all been successfully knocked out using this method. Each of these mutants has also been evaluated as a vaccine candidate as discussed in the next section.

\section{PREVIOUS MAP VACCINE STUDIES}

MAP vaccination studies have been thoroughly summarized for cattle, sheep and goats by examining pathological, epidemiological and production effects of vaccination (Bastida and Juste, 2011). This meta-analysis revealed that while vaccination is useful in limiting microbial contamination and production losses, most of the vaccines are similar in content and preparation. These findings have recently been reinforced by the reduction of fecal contamination in three dairy herds using a killed vaccine (Knust et al., 2013). The possibility exists that new generations of vaccine formulations, including live attenuated, may further improve on the killed vaccine preparations currently being used. This section summarizes the pertinent literature on MAP vaccination as it relates to the JDIP trials.

Subunit vaccines have been delivered using heterologous hosts such as Salmonella (Chandra et al., 2012), or Lactobacillus (Johnston et al., 2014). Only two DNA vaccines against MAP have been tested in mice. One used expression library DNA immunization of Balb/cJ mice and challenged with a recently isolated bovine MAP strain 6112 (Huntley et al., 2005). Four pools of 108 clones each protected mice from colonization of spleen and mesenteric lymph nodes. The second study tested a cocktail of five genes encoding antigen $85 \mathrm{~A}, \mathrm{~B}$, and $\mathrm{C}$ along with superoxide dismutase and $35-\mathrm{kDa}$ protein by intramuscular injection of C57/BL6 (Park et al., 2008b). There was a significant reduction in colonization of the liver and spleen of mice immunized with this 
cocktail. The subunit vaccines from both studies induced a Th1 immune response as measured by interferon gamma; however, none of these DNA formulations were tested in cattle or other ruminant hosts.

Several MAP vaccine candidates have been analyzed in isolation making it difficult to directly compare candidates between studies. Some have been bench marked against a commercially available vaccine (Faisal et al., 2013a; Hines et al., 2014) while other studies lack even that as a reference. As mentioned, all commercial vaccine formulations consist of a killed whole-cell preparation of MAP in an oil emulsion, but there are many other types of vaccines. Still other vaccine formulations included live M. avium bacteria, but it is not clear if or how they were attenuated (Begg and Griffin, 2005). In the current JDIP vaccine trial, we took a different approach in identifying a viable, yet attenuated vaccine strain, reasoning that a live strain will maintain a proinflammatory Th1 response (Stabel, 2000; Coussens, 2004). Th1associated cytokines including interferon gamma, interleukin-2 (IL-2), IL-12 and tumor necrosis factor alpha were measured after antigen stimulation of cultured peripheral blood monocytes in these studies.

While there are no published bovine vaccine trials in Johne's disease, there are a number of caprine and ovine vaccine trials. Furthermore, a number of MAP vaccine studies in mice have been reported, but they are too numerous to adequately be summarized here. Importantly, only a few studies that began using the mouse model were further evaluated in a ruminant host (Scandurra et al., 2010; Park et al., 2011) and none using the number of mutant strains in the JDIP trials. This was a key component in the design of the JDIP vaccine trial. Using the data described in this special topics issue, we can now assess the predictive value of the mouse trial in obtaining good candidates for the ruminant host.

\section{ATTENUATED MUTANTS IN THE JDIP VACCINE STUDY}

What was known about the mutants prior to enrollment in the JDIP vaccine study? Some of the mutants remain unpublished or have only recently been submitted for publication, however, others have been described prior to the JDIP trials. Information concerning all mutants included in the JDIP trials is summarized in Table 1. The first MAP mutants created by allelic exchange produced directed knockouts of $l s r 2$, relA, and $p k n G$ mutants in 2008 (Park et al., 2008a) and all three were included in the JDIP trial (Table 1). These three genes were selected based on the virulence properties of their orthologs in Mycobacterium tuberculosis and Mycobacterium bovis. Lsr2 is a cytosolic protein implicated in cell wall lipid biosynthesis and antibiotic resistance in M. smegmatis (Chen et al., 2006; Colangeli et al., 2007). Protein kinase $\mathrm{G}$, encoded by $p k n G$, is secreted by $M$. tuberculosis and M. bovis within the phagosome of macrophages and is thought to inhibit phagolysosomal fusion (Walburger et al., 2004). RelA in M. tuberculosis is involved in the stringent response that is activated in nutrient limiting conditions. Specifically, RelA in M. tuberculosis synthesizes the hyperphosphorylated guanine nucleotides that accumulate in nutrient limiting conditions and inactivating this gene severely reduced long-term survival in mice (Dahl et al., 2003).
Two of these MAP mutants, $\Delta p k n G$ and $\Delta r e l A$, were later tested as vaccine candidates in cultured macrophages, calves and kid goats (Park et al., 2011). Although both were attenuated in bovine macrophages compared to the wild-type strain on day 6 , it was shown that $\triangle r e l A$ was the better vaccine candidate since no MAP was found in the tissues of calves vaccinated with the relA mutant. Furthermore, vaccination with the $p k n G$ mutant did not inhibit challenge with MAP in the kid goats as the challenge bacteria were present in high numbers in all 9 tissues evaluated, whereas the relA mutant vaccinates were free of the challenge inoculum in 8 of the 9 tissues taken from kid goats (Park et al., 2011). However, both mutants induced effector memory $\mathrm{T}$ cells similar to the wild type strain. Although $\Delta l s r 2$ was included in the study, further experiments were needed to assess the in vivo survival of $\Delta l s r 2$ because of fungus problems in readout cultures (see Park et al., 2014, this issue). Of interest, a second mutant containing an $l s r 2$ transposon insertion was developed by another laboratory and included in the JDIP study and was coded 317 (Table 1). A report on this mutant has just been submitted publication, and it was evaluated in the mouse challenge model in phase II but not the phase III JDIP trial. Another interesting observation was that three independent investigators submitted mutants with insertions in the same gene, MAP1566, which encodes a hypothetical protein (Li et al., 2005). This is surprising because the MAP genome contains over 4300 targets for transposon insertion, yet of the 22 mutants enrolled in this study 3 are in the same target (MAP1566) and another two are in $l s r 2$.

One of the three $\triangle$ MAP1566 mutants along with the ppiA mutant, coded as 322 and 323, respectively, in Table 1, were analyzed in macrophages, mice, and goats (Scandurra et al., 2010). All three model systems demonstrated that $\triangle \mathrm{MAP} 1566$ was more attenuated than the ppiA mutant. However, macrophages produced less IL-10 when infected with the ppiA mutant and it persisted longer in mice. In the goat experiment, the challenge strain was not cultured from any of the tissues in goats vaccinated with $\triangle$ MAP1566, whereas $15 \%$ of the tissues taken from the $\triangle p p i A$ immunized goats were positive by Bactec $12 \mathrm{~B}$ culture for the challenge strain (Scandurra et al., 2010). Collectively, these data suggest that the $\triangle \mathrm{MAP} 1566$ strain is a better vaccine than $\triangle p p i A$.

Other mutants that were shown to be attenuated in mice prior to testing in the JDIP vaccine trial include $k d p C$, $p s t A, u m a A 1$, and fabG2_2 (Shin et al., 2006). The pstA gene encodes a large $(12 \mathrm{~kb})$ non-ribosomal synthetase protein involved in glycopeptidolipid biosynthesis and was later shown to contribute to biofilm formation and invasion of the calf intestine (Wu et al., 2009). The umaA1 gene codes for a mycolic acid synthase that was studied in M. tuberculosis (Yuan et al., 1995). The $k d p C$ mutant showed significantly lower colonization levels in the liver and intestine of mice at all time points, compared to the wild-type and also displayed less granulomatous inflammation (Shin et al., 2006). The pstA, umaA1, and fabG2_2 mutants showed reduced bacterial colonization of the mouse intestine at later time points. Finally, a manuscript is in preparation describing the mutants constructed at the University of Nebraska (Table 1). Among this group are transposon insertions between genes (intergenic) rather than within a gene. 
Table 1 | Transposon mutant vaccine candidates of MAP enrolled in the JDIP vaccine trials.

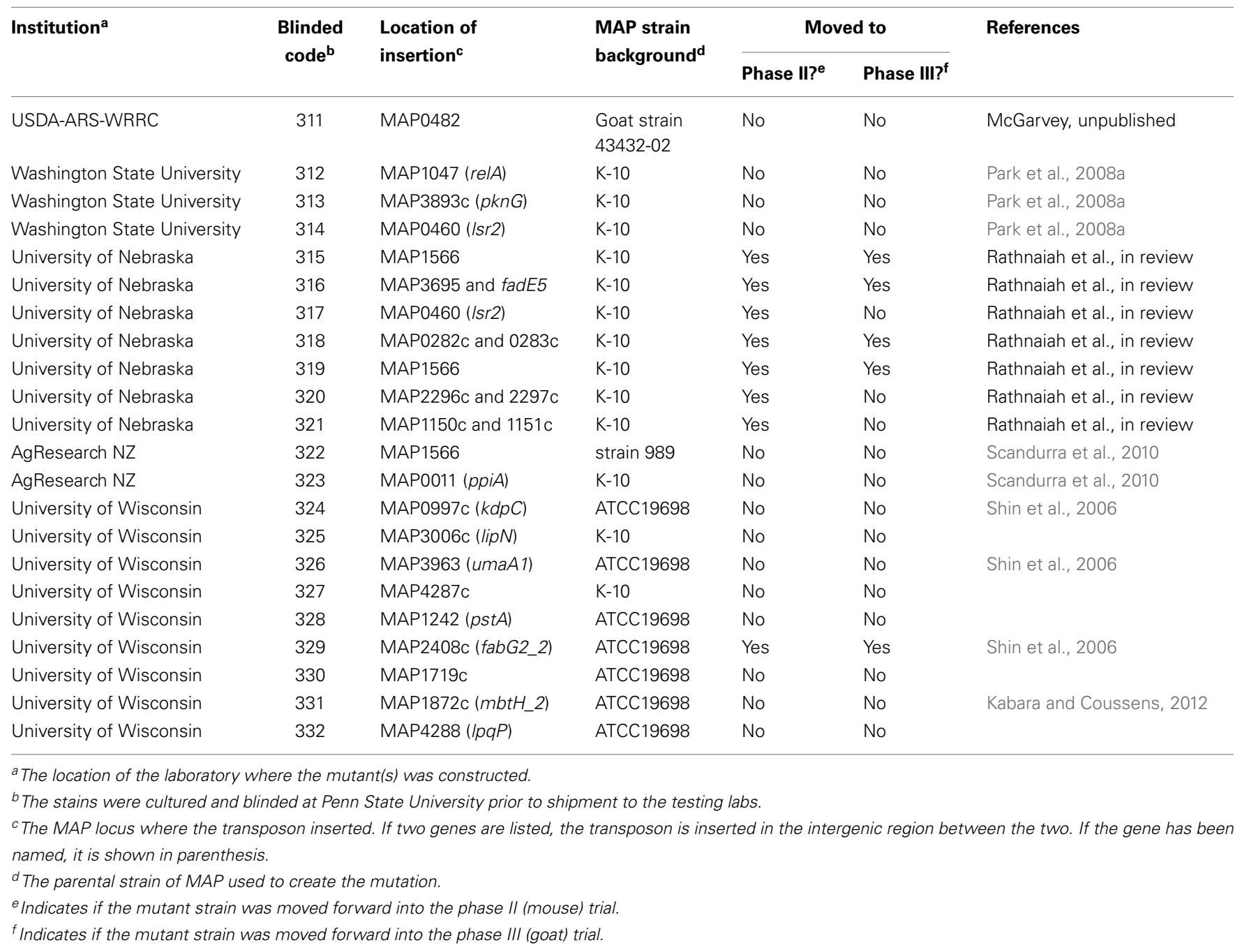

A subset of the mutants in the JDIP study was examined for ability to regulate host cell apoptosis of macrophages (Scandurra et al., 2010; Kabara and Coussens, 2012). Apoptosis is an important tool in the fight against mycobacterial infections as apoptotic bodies are taken up by other antigen presenting cells. Mycobacteria contained in apoptotic bodies are destroyed and their antigens presented to immune cells. The lsr 2 knockout was most similar to the wild-type in terms of controlling apoptosis among the mutants tested, indicating that $l s r 2$ is not involved in this process. However, a Tn5367 insertion in MAP1872c was not able to prevent apoptosis, indicating that this gene may be involved in regulating this host cell process. The gene encodes an iron acquisition protein (Zhu et al., 2008), which is an important process for MAP survival in the host. The other mutants tested were not significantly different from the uninfected control, which demonstrated the level of spontaneous apoptosis in culture. A second study examined apoptosis in bovine macrophages (Scandurra et al., 2010) and two of the mutants overlapped between that study and the Kabara and Coussens study. These mutants included the ppiA knockout (code
323 in Table 1) and MAP1566 (code 322). While neither of these mutants demonstrated control of apoptosis in the Kabara and Coussens study, $\triangle$ MAP1566 showed a significant reduction in apoptosis compared to the wild type strain in the Scandurra et al. study. The ppiA mutant was inconclusive since macrophages from the two cows examined gave divergent results (Scandurra et al., 2010). When tested independently all these mutants showed promise as a vaccine candidate for Johne's disease. So we developed a strategy to directly compare all of these mutants in the same vaccine trial through the JDIP research consortium.

\section{INTERFERENCE WITH DIAGNOSTIC TESTING FOR TB OR JOHNE'S DISEASE}

Although it has been suggested there might be an added benefit to vaccinate against MAP for cross protection with TB (Perez De Val et al., 2012), it is generally seen as a negative due to interference with diagnostic testing for $\mathrm{TB}$, a highly regulated animal disease. In fact, a primary reason why whole cell vaccines against Johne's disease are not routinely used in the United States is due to the interference with diagnostic tests for both Johne's disease 
and particularly bovine TB. This same reason is why vaccination was also reserved only for farms in the Netherlands with high incidence of Johne's disease (Muskens et al., 2002). Immunization with Mycopar ${ }^{\circledR}$ will interfere with Johne's diagnostic tests, particularly the IFN- $\gamma$ test, but not with the comparative cervical skin test which was $100 \%$ specific within the first year of vaccination (Stabel et al., 2011). In addition, M. bovis serological tests, which included the ESAT-6, CFP-10, and MPB83 antigens, were also negative in MAP vaccinated calves. Likewise, an immediate strong and long-lasting MAP-specific IFN- $\gamma$ response was observed in two herds vaccinated with a heat-killed vaccine (Muskens et al., 2002). Caution must be used if administering Gudair ${ }^{\circledR}$ to cattle since it appears to lower the sensitivity of the bovine TB skin test (Coad et al., 2013). Dairy herds vaccinated with Silirum ${ }^{\circledR}$ showed that the comparative intradermal test (CIT) had low cross reactivity; however the single bovine intradermal tubculin test would result in 5-6\% false positive results for TB (Garrido et al., 2013). These results are similar with MAP vaccinated goats (Chartier et al., 2012). Thus, the CIT test can be considered useful if evaluating TB in a MAP vaccinated herd. This finding paves the way for live attenuated vaccines for MAP. Perhaps even more encouraging are the novel biomarkers identified from proteomics of both the host and pathogen that suggest peptides in circulation may overcome the issue of cross-reactivity (Seth et al., 2009). However, even if producers had to deal with a low level of false positive results for TB infection, we believe that if a strong live vaccine were developed that could prevent infection and enable effective disease control, the vaccine would be widely implemented.

\section{CONCEPTION OF THE JDIP VACCINE TRIAL}

It was against this background that the idea for establishing independent testing labs to evaluate all the available vaccine candidates was conceived. This multi-institutional study was based on the following principles. Through the formation of a research consortium on Johne's disease, which was funded by the USDANIFA from 2002 to 2011, this governing body was successful in setting up a world-wide search for the best performing vaccine candidates. The idea behind this project was to combine the resource and intellect of JDIP with the financial resource of USDA-APHIS-VS to identify the best possible attenuated vaccine available. On January 12, 2008 a meeting was held in Chicago, Illinois to devise a coordinated effort to test attenuated vaccine candidates submitted by Johne's disease researchers. Although a few vaccine trials have tested individual mutants in macrophages, mice and goats, no trial of this scope using 5 or more mutants tested in parallel has ever been performed.

\section{TRIAL DESIGN}

To begin, investigators around the world were asked to submit their best-attenuated mutants, along with any efficacy data generated in their labs, to the JDIP study. A multi-institutional universal material transfer agreement was drafted and finalized enabling all investigators to submit their vaccine candidate(s) to Penn State University (PSU) while at the same time, protecting intellectual property rights of the investigator. PSU cultured each strain to the specified optical density and coded each strain for blinding before sending onto specific labs to perform the efficacy trials.

Three gates were decided upon which the mutants had to pass through at each phase of the study. The first gate measured survival of mutants in primary bovine macrophages, where the most attenuated strains were considered the best candidates. The second gate was protection from colonization by challenge bacteria in the liver and spleen of mice and the third gate was protection from colonization using the goat challenge model (Hines et al., 2007). A triage process had to be implemented at each gate due to funding limitations, but we acknowledge it would have been ideal to test all the vaccine strains in all three trials.

Five investigators from the United States and New Zealand responded to the JDIP request and submitted their attenuated MAP mutants. It is noteworthy that directed or random knockouts in MAP are not trivial to obtain and hence, only a few investigators had possession of such mutants. A total of 22 transposon-marked mutants were submitted (Table 1). Interestingly, among the 22 mutants, a few had insertions in the same gene, but were submitted by different investigators. As mentioned above, MAP1566 insertions were present in 3 strains, 2 of them from the same lab contained insertions in different locations within the gene. Also 2 mutants submitted by independent labs were MAP0460 knockouts. Given the number of potential gene targets in this organism $(>4300)$, this duplication was unexpected, but provided some interesting built-in comparisons.

\section{VACCINE CANDIDATE SELECTION}

The initial criteria for candidate vaccine selection were based on data obtained by the investigator who constructed the mutant. Each investigator determined independently the suitability of their mutant. In some cases these results were published, but regardless, the net was cast broad and wide to obtain as many MAP mutants as possible for testing. However, there were some minimal qualifications. To enter the first trial each mutant (1) must be viable in vitro (no sonicated extracts or heat-inactivated preps) and (2) growth rate in broth cultures must be similar to wild-type MAP. The mutant strains were sent to PSU under a unique pan-institutional material transfer agreement that allowed the submitting investigators to retain full intellectual property. Each mutant strain received was tested for culture, coded to blind testing labs and quality control tested prior to shipping to the testing labs. For example, the PSU lab confirmed a lack of contamination in the culture by PCR and plating on blood agar and/or BHI before shipping. Therefore, after receiving the strains, at least 60 days were needed to prepare each strain for shipment. Five submitted strains were not shipped to the phase I testing labs for the following reasons. One showed incredibly slow and anemic growth in both liquid and solid culture, regardless of several attempts. For two others, the reference colony forming units (CFU) was not obtainable by the end of phase I. And the remaining two arrived after the due date for submission. Although PSU started to grow them regardless, there was not enough time to include them in this initial trial. A general summary of each trial is listed below. 


\section{PHASE I: SURVIVAL IN PRIMARY BOVINE MACROPHAGES}

The single goal of this experiment was to test survival in primary bovine macrophages because attenuation in this environment can infer potential vaccine candidates. Primary bovine macrophages were cultured and infected in two laboratories, one at the University of Minnesota (UMN) and the other at the University of Wisconsin (UW). In order for these labs to start their macrophage studies, a final CFU number was needed from PSU to infect at the correct multiplicity of infection. Once the cells reached $\mathrm{OD}_{600}=0.50$, the PSU lab determined the reference CFU and started to regrow the strains for shipment. The manuscript describing the results of this study was just published (Lamont et al., 2014).

Colony counts from cultures over time in macrophages were obtained to give the slope (growth over time). Basically, a negative slope demonstrated attenuation because it meant there were less viable bacteria recovered from bovine macrophages as time increased (Lamont et al., 2014). When examining the data by slope alone, it was evident which mutants had a negative slope and which did not, but when looking at the individual CFU data, it was clear that considering slope alone was misleading. Therefore, JDIP solicited the Michigan State University (MSU) lab to perform an apoptosis study of the mutants. Some mutants were not included in this study because of the staggered nature in which they were received or the lack of permission to include the mutant strain in a publication. Most of the strains were sent to the MSU lab for the apoptosis experiment (Kabara and Coussens, 2012). Some of the mutants arrived several months late, and at that point, JDIP needed the data from the study to help make a decision on which mutant strains to go forward in the mouse trial.

Results of the experiments from each trial were sent directly to Cornell University for data analysis. Slope of the growth in macrophages was used to compare mutants. The results showed subtle changes in survival of the mutant strains (manuscript in preparation). Once the analysis was complete and decisions made about which strains would advance to the next phase, the blind was decoded and recoded for the next experiment.

\section{PHASE II: THE MOUSE TRIAL}

This experiment was designed to test the remaining 8 attenuated vaccine strains in the mouse challenge model. The readout was colonization (CFU) of the liver and spleen from vaccinated and control mice (Bannantine et al., 2014). Silirum ${ }^{\circledR}$ was the commercial vaccine run in parallel for this trial. Each mouse received $10^{5} \mathrm{CFU}$ of the live attenuated vaccine strain in $0.5 \mathrm{ml}$ PBS by intraperitoneal injection.

The persistence of the vaccine candidates was measured at 6, 12 , and 18 weeks post-vaccination. Only strains 320, 321, and 329 colonized both the liver and spleens up until the 12 -week time point. The remaining five mutants showed no survival in those tissues, indicating their complete attenuation in the mouse model. The vaccine strains demonstrated different levels of protection based on MAP colonization in liver and spleen tissues at 12 and 18 weeks post-vaccination. Based on total MAP burden in both tissues at both time points, strain 315 (MAP1566::Tn5370) was the most protective whereas strain 318 (intergenic Tn5367 insertion between MAP0282c and MAP0283c) had the most colonization (Bannantine et al., 2014). Mice vaccinated with an undiluted commercial vaccine preparation displayed the strongest antibody responses as well as enlarged spleens.

The effect of persistence of the three vaccine strains in mice is unclear. However, it is clear from the goat trial described below that the vaccine strains did not persist indefinitely as only the challenge strain was identified by PCR analysis at the 2 month time point (Hines et al., 2014). It would have been interesting to see if the $p k n G$ mutant was persistent in the JDIP goat study, but that mutant was not included in phase III of the trial due to triage after the macrophage study (Lamont et al., 2014). Significantly the relA mutant, that also showed attenuation in macrophages, did not make it through to the last phases of the trial, even though a preliminary study in goats showed it was immune eliminated. The immune response elicited by the mutant limited infection with MAP under experimental challenge conditions (Park et al., 2011). A further study conducted in parallel with the JDIP attenuated vaccine studies, but using calves, has extended the findings in goats with similar results (Park et al., 2014).

\section{PHASE III: THE GOAT TRIAL}

Data from this final trial in a ruminant host were used to rank the remaining vaccine candidates to determine the best attenuated vaccine. Logistic issues interfered with testing and two strains that showed promising results in mice (strains 320 and 321) were not evaluated in goats (Bannantine et al., 2014). A second goal of this phase III vaccine trial was to validate the goat challenge model originally proposed in 2007 (Hines et al., 2007). Trial was performed at the University of Georgia-Tifton Veterinary Diagnostic and Investigational Laboratory.

Eighty 2-month-old goat kids were separated into 8 groups with 10 kids in each group. Three groups were dedicated to controls (wild-type MAP K-10, PBS and Silirum ${ }^{\circledR}$ vaccine). The remaining 5 groups consisted of the MAP vaccine candidates. The animals in each group were housed in identical treatment rooms in a manner to reduce "pen effect." Baseline blood and fecal samples were taken at the beginning of the study. As with the other trials, the investigators were blinded as to the identity of the experimental vaccines. The Silirum ${ }^{\circledR}$ vaccine was administered as a single dose and used according to manufacturer instructions. The study length was approximately 18 months with a full 12month period post-challenge. Two doses of each mutant vaccine were administered as described (Hines et al., 2014). All vaccine and challenge doses were delivered orally by syringe in pasteurized commercial goat milk.

While none of the vaccines tested prevented MAP infection or eliminated fecal shedding in goats, the $\Delta f a b G 2 \_2$ vaccine strain (coded 329 in Table 1) did lower the incidence and severity of infection as measured by lesion score, tissue pathology, fecal culture and fecal PCR (Hines et al., 2014). The fabG2_2 gene encodes a 3-ketoacyl reductase of MAP and the transposon is inserted in the C-terminal half of the gene (Settles et al., 2014). This mutant has recently been shown to have defects in intestinal and liver persistence (Settles et al., 2014). Additional preliminary studies suggest that this mutation may not be stable as it could not be confirmed by PCR analysis. The $\triangle f a b G 2 \_2$ vaccine strain did 
not outperform the commercial vaccine control in the goat trial. This result may be explained by delivery route of the attenuated strains vs. the commercial vaccine. Attenuated strains were delivered orally and the commercial vaccine was delivered subcutaneously. Ideally, the route of vaccination should have been identical, but a second objective was to validate the goat challenge model proposed by the AMSC (Hines et al., 2007). In hindsight, the route of vaccination should have been the same for all vaccines.

\section{CONCLUSIONS}

Vaccination against Johne's disease has considerable potential as a key management tool to control disease and transmission in ruminant livestock. Specifically, the use of attenuated mutants provide several advantages that include stimulation of a broad cellular immune response, ease of delivery, built in adjuvant characteristics and comparative cost. Several lessons were learned from the three-phase JDIP vaccine project as discussed below, and provide a rational framework for testing future vaccines against Johne's disease.

First, the results suggest that single gene knockout strains may not represent optimal vaccine candidates for MAP given the need to satisfy the "Goldilocks rule"-where the level of attenuation has to be just right in order to survive or replicate in target cells and stimulate a protective immune response but without causing disease. Consistent with this, while MAP vaccine candidates evaluated in the current trials were single gene knockouts, recent studies showed that a double mutant constructed in $M$. tuberculosis $(\triangle f b p A \Delta s a p M$ was highly attenuated in human macrophages and, intriguingly, more immunogenic than the single knockout $(\triangle f b p A)$ (Saikolappan et al., 2012). These data also suggest the importance of careful targeting of genes/proteins for deletion based on prior knowledge of their role in pathogenesis, immunogenicity, or pathogen survival in vivo. For instance, the rationale for construction of the $M$. tuberculosis ( $\triangle f b p A \Delta s a p M$ strain was that $\triangle f b p A$, which lacks Ag85, had already demonstrated immunogenicity and protection in mice, but the additional deletion of the acid phosphatase gene $(\Delta s a p M)$ enabled the host phagosome to mature (Saikolappan et al., 2012). A considered approach for MAP vaccine candidate development that target orthologs in $M$. tuberculosis that have shown promise in vaccine efficacy trials or are known to be highly immunogenic targets seems reasonable, but has its limitations. For instance, there is no corresponding ortholog to sapM in MAP (Li et al., 2005), and hence, construction of a double knockout involving that locus would not be possible.

We note that the MAP vaccines evaluated herein represented the most comprehensive screen of MAP attenuated mutants to date, and included vaccine candidates with prior evidence of attenuation with in vitro or in vivo model systems that were enrolled on a volunteer basis (i.e., investigators were broadly invited and those that volunteered to participate and submit candidate vaccines were enrolled). This appears a reasonable strategy to adopt since it provides a facile and transparent mechanism for benchmarking candidate vaccines to each other to help identify the most promising candidates. However, not all described or available candidate strains of MAP were enrolled in this trial.
Notably, the recently described $\Delta l e u D$, which provides protection in a goat model of infection (Faisal et al., 2013a) was not submitted for evaluation in the current trials, and neither were a set of invasion mutants (Alonso-Hearn et al., 2008) which should be included in future investigations.

Our studies have highlighted several major lacuna in our understanding of in vitro correlates of vaccine immunity and strain attenuation in MAP. For instance, how long should a strain of MAP take to be killed by phagocytes to be considered attenuated? And what are the physiologic (e.g., doubling time) or immunological (e.g., cytokine profiles elicited) correlates of protective immunity for MAP that should be considered prior to advancing a vaccine candidate? These and other similar questions regarding in vitro correlates of protection remain and need to be the focus of future investigations.

Second, and perhaps more importantly, the rationale for the use of the macrophage-to-mouse-to-native host pipeline for MAP vaccine development needs to be reconsidered. The survival of MAP strains in cultured macrophages has been previously used as a criterion for the selection of candidate vaccines for further testing in animal models. For instance, transposon mutants of MAP that were attenuated in macrophages were also shown to be attenuated in mice (Scandurra et al., 2009). While this strategy has been applied for selection of vaccine candidates for numerous other pathogens, particularly those that impact humans, the lack of validated or well rationalized in vitro correlates of protection does not enable an accurate assessment of either attenuation or immunogenicity of a given candidate in vivo. This shortcoming is further exacerbated by the lack of context for the host immune system and the natural milieu, and the fact that only short-term attenuation can be measured in macrophages, even though these are long-lived cells in vivo, can remain viable for only 48 -h or so in laboratory culture. And can only measure degrees of attenuation in a somewhat artificial system that does not predict protection (Lamont et al., 2014).

The results of our investigations raise important questions on whether mice are relevant or appropriate models for assessment of anti-MAP vaccines. It has been long recognized that the mouse is not a natural host for MAP, but rather employed as a model for cost and practical (e.g., availability of immunologic reagents) considerations that may not be of relevance to infection or immunity against MAP in the natural host. By not being a natural host, challenge and vaccine studies are made difficult since it is often not possible to consistently infect mice. Furthermore, culturing of MAP from intestinal tissues (the predominant site of infection in the natural ruminant host) is rarely achieved in mice, and successfully infected animals do not exhibit any of the typical clinical signs (e.g., diarrhea) that are associated with MAP infection in ruminants. In hindsight, this is not altogether surprising since MAP infection is primarily a chronic granulomatous infection of the intestinal tract, and given that recent studies suggest that the mouse is a poor model for inflammatory conditions in humans (Seok et al., 2013), mice are not very likely to represent good models for chromic inflammatory diseases such as JD in cattle either. This is also consistent with recent observations (Scandurra et al., 2010; Park et al., 2011) that suggest attenuation in macrophages and marginal protection in the mouse model may 
not be a good predictor for the ruminant host. In addition, it was previously noted that a lack of survival in bovine macrophages does not predict survival in goats. For instance, the $p k n G$ mutant was attenuated in macrophages and yet showed persistence in goats and the immune response elicited by the mutant did not affect colonization by the MAP challenge (Park et al., 2011).

Thankfully, unlike the situation in humans, Johne's disease investigators have the luxury working with the natural (ruminant) host despite the apparent high costs. We suggest that the ability to quickly and relatively inexpensively use macrophage/mouse models, though tempting, may represent a situation of being "penny wise and pound foolish" and in the absence of clearly articulated and validated correlates of immune protection in these models, may result in perfectly good candidates being discarded during the triage process or, as was observed in the studies described herein, apparently promising targets move forward at considerable expense, only to fail to perform as expected when evaluated in the natural host.

In sum, the results of our studies strongly suggest that future investigations of MAP vaccine candidates are best conducted in the natural host, and considerable efficiencies can be realized by using a coordinated approach and standardized protocols for comparative benchmarking and evaluation of MAP vaccine candidates.

\section{ACKNOWLEDGMENTS}

This study was supported by the USDA-CAP program entitled the Johne's Disease Integrated Program and also by USDAAPHIS-Veterinary Services. We also thank the testing institutions against which some resources were leveraged. No endorsements of reagents or equipment are implied in these studies. Special thanks are given for the expert technical assistance of Robab Katani and Lingling Li who performed many behind the scenes experiments to keep these trials moving along.

\section{REFERENCES}

Achkar, J. M., and Casadevall, A. (2013). Antibody-mediated immunity against tuberculosis: implications for vaccine development. Cell Host Microbe 13, 250-262. doi: 10.1016/j.chom.2013.02.009

Alonso-Hearn, M., Patel, D., Danelishvili, L., Meunier-Goddik, L., and Bermudez, L. E. (2008). The Mycobacterium avium subsp. paratuberculosis MAP3464 gene encodes an oxidoreductase involved in invasion of bovine epithelial cells through the activation of host cell Cdc42. Infect. Immun. 76, 170-178. doi: 10.1128/IAI.01913-06

Bannantine, J. P., Everman, J. L., Rose, S. J., Babrak, L., Katani, R., Barletta, R. G., et al. (2014). Evaluation of eight live attenuated vaccine candidates for protection against challenge with virulent Mycobacterium avium subspecies paratuberculosis in mice. Front. Cell. Infect. Microbiol. 4:88. doi: 10.3389/fcimb.2014. 00088

Bastida, F., and Juste, R. A. (2011). Paratuberculosis control: a review with a focus on vaccination. J. Immune Based Ther. Vaccines 9:8. doi: 10.1186/1476-8518-9-8

Begg, D. J., and Griffin, J. F. (2005). Vaccination of sheep against $M$. paratuberculosis: immune parameters and protective efficacy. Vaccine 23, 4999-5008. doi: 10.1016/j.vaccine.2005.05.031

Bull, T. J., Schock, A., Sharp, J. M., Greene, M., Mckendrick, I. J., Sales, J., et al. (2013). Genomic variations associated with attenuation in Mycobacterium avium subsp. paratuberculosis vaccine strains. BMC Microbiol. 13:11. doi: 10.1186/1471-2180-13-11

Cavaignac, S. M., White, S. J., De Lisle, G. W., and Collins, D. M. (2000). Construction and screening of Mycobacterium paratuberculosis insertional mutant libraries. Arch. Microbiol. 173, 229-231. doi: 10.1007/s002039900132
Chandra, S., Faisal, S. M., Chen, J. W., Chen, T. T., Mcdonough, S. P., Liu, S., et al. (2012). Immune response and protective efficacy of live attenuated Salmonella vaccine expressing antigens of Mycobacterium avium subsp. paratuberculosis against challenge in mice. Vaccine 31, 242-251. doi: 10.1016/j.vaccine.2012.09.024

Chartier, C., Mercier, P., Pellet, M. P., and Vialard, J. (2012). Effect of an inactivated paratuberculosis vaccine on the intradermal testing of goats for tuberculosis. Vet. J. 191, 360-363. doi: 10.1016/j.tvjl.2011.03.009

Chen, J. M., German, G. J., Alexander, D. C., Ren, H., Tan, T., and Liu, J. (2006). Roles of Lsr2 in colony morphology and biofilm formation of Mycobacterium smegmatis. J. Bacteriol. 188, 633-641. doi: 10.1128/JB.188.2.633-6 41.2006

Chen, J. W., Faisal, S. M., Chandra, S., Mcdonough, S. P., Moreira, M. A., Scaria, J., et al. (2012). Immunogenicity and protective efficacy of the Mycobacterium avium subsp. paratuberculosis attenuated mutants against challenge in a mouse model. Vaccine 30, 3015-3025. doi: 10.1016/j.vaccine.2011.11.029

Chen, L. H., Kathaperumal, K., Huang, C. J., Mcdonough, S. P., Stehman, S., Akey, B., et al. (2008). Immune responses in mice to Mycobacterium avium subsp. paratuberculosis following vaccination with a novel $74 \mathrm{~F}$ recombinant polyprotein. Vaccine 26, 1253-1262. doi: 10.1016/j.vaccine.2007.12.014

Chiodini, R. J., and Van Kruiningen, H. J. (1986). The prevalence of paratuberculosis in culled New England cattle. Cornell Vet. 76, 91-104.

Coad, M., Clifford, D. J., Vordermeier, H. M., and Whelan, A. O. (2013). The consequences of vaccination with the Johne's disease vaccine, Gudair, on diagnosis of bovine tuberculosis. Vet. Rec. 172, 266. doi: 10.1136/vr.101201

Colangeli, R., Helb, D., Vilcheze, C., Hazbon, M. H., Lee, C. G., Safi, H., et al. (2007). Transcriptional regulation of multi-drug tolerance and antibioticinduced responses by the histone-like protein Lsr2 in M. tuberculosis. PLoS Pathog. 3:e87. doi: 10.1371/journal.ppat.0030087

Coussens, P. M. (2004). Model for immune responses to Mycobacterium avium subspecies paratuberculosis in cattle. Infect. Immun. 72, 3089-3096. doi: 10.1128/IAI.72.6.3089-3096.2004

Dahl, J. L., Kraus, C. N., Boshoff, H. I., Doan, B., Foley, K., Avarbock, D., et al. (2003). The role of RelMtb-mediated adaptation to stationary phase in longterm persistence of Mycobacterium tuberculosis in mice. Proc. Natl. Acad. Sci. U.S.A. 100, 10026-10031. doi: 10.1073/pnas.1631248100

Faisal, S. M., Chen, J. W., Yan, F., Chen, T. T., Useh, N. M., Yan, W., et al. (2013a). Evaluation of a Mycobacterium avium subsp. paratuberculosis leuD mutant as a vaccine candidate against challenge in a caprine model. Clin. Vaccine Immunol. 20, 572-581. doi: 10.1128/CVI.00653-12

Faisal, S. M., Yan, F., Chen, T. T., Useh, N. M., Guo, S., Yan, W., et al. (2013b). Evaluation of a Salmonella vectored vaccine expressing Mycobacterium avium subsp. paratuberculosis antigens against challenge in a goat model. PLoS ONE 8:e70171. doi: 10.1371/journal.pone.0070171

Foley-Thomas, E. M., Whipple, D. L., Bermudez, L. E., and Barletta, R. G. (1995). Phage infection, transfection and transformation of Mycobacterium avium complex and Mycobacterium paratuberculosis. Microbiology 141(Pt 5), 1173-1181. doi: 10.1099/13500872-141-5-1173

Garrido, J. M., Vazquez, P., Molina, E., Plazaola, J. M., Sevilla, I. A., Geijo, M. V., et al. (2013). Paratuberculosis vaccination causes only limited cross-reactivity in the skin test for diagnosis of bovine tuberculosis. PLoS ONE 8:e80985. doi: 10.1371/journal.pone.0080985

Harris, N. B., Feng, Z., Liu, X., Cirillo, S. L., Cirillo, J. D., and Barletta, R. G. (1999), Development of a transposon mutagenesis system for Mycobacterium avium subsp. paratuberculosis. FEMS Microbiol. Lett. 175, 21-26.

Hines, M. E., 2nd, Stabel, J. R., Sweeney, R. W., Griffin, F., Talaat, A. M., Bakker, D., et al. (2007). Experimental challenge models for Johne's disease: a review and proposed international guidelines. Vet. Microbiol. 122, 197-222. doi: 10.1016/j.vetmic.2007.03.009

Hines, M. E., 2nd, Turnquist, S. E., Ilha, M. R. S., Rajeev, S., Jones, A. L., Whittington, L., et al. (2014). Evaluation of novel oral vaccine candidates and validation of a caprine model of Johne's disease. Front. Cell. Infect. Microbiol. 4:26. doi: 10.3389/fcimb.2014.00026

Hostetter, J. M., Steadham, E. M., Haynes, J. S., Bailey, T. B., and Cheville, N. F. (2002). Cytokine effects on maturation of the phagosomes containing Mycobacteria avium subspecies paratuberculosis in J774 cells. FEMS Immunol. Med. Microbiol. 34, 127-134. doi: 10.1111/j.1574-695X.2002.tb00613.x

Huntley, J. F., Stabel, J. R., Paustian, M. L., Reinhardt, T. A., and Bannantine, J. P. (2005). Expression library immunization confers protection against 
Mycobacterium avium subsp. paratuberculosis infection. Infect. Immun. 73, 6877-6884. doi: 10.1128/IAI.73.10.6877-6884.2005

Johnston, C. D., Bannantine, J. P., Govender, R., Endersen, L., Pletzer, D., Weingart, H., et al. (2014). Enhanced expression of codon optimized Mycobacterium avium subsp. paratuberculosis antigens in Lactobacillus salivarius. Front. Cell. Infect. Microbiol. 4:120. doi: 10.3389/fcimb.2014.00120

Kabara, E., and Coussens, P. M. (2012). Infection of primary bovine macrophages with Mycobacterium avium subspecies paratuberculosis suppresses host cell apoptosis. Front. Microbiol. 3:215. doi: 10.3389/fmicb.2012.00215

Kalis, C. H., Hesselink, J. W., Barkema, H. W., and Collins, M. T. (2001). Use of long-term vaccination with a killed vaccine to prevent fecal shedding of Mycobacterium avium subsp paratuberculosis in dairy herds. Am. J. Vet. Res. 62, 270-274. doi: 10.2460/ajvr.2001.62.270

Kathaperumal, K., Kumanan, V., Mcdonough, S., Chen, L. H., Park, S. U., Moreira, M. A., et al. (2009). Evaluation of immune responses and protective efficacy in a goat model following immunization with a coctail of recombinant antigens and a polyprotein of Mycobacterium avium subsp. paratuberculosis. Vaccine 27, 123-135. doi: 10.1016/j.vaccine.2008.10.019

Kathaperumal, K., Park, S. U., Mcdonough, S., Stehman, S., Akey, B., Huntley, J., et al. (2008). Vaccination with recombinant Mycobacterium avium subsp. paratuberculosis proteins induces differential immune responses and protects calves against infection by oral challenge. Vaccine 26, 1652-1663. doi: 10.1016/j.vaccine.2008.01.015

Knust, B., Patton, E., Ribeiro-Lima, J., Bohn, J. J., and Wells, S. J. (2013). Evaluation of the effects of a killed whole-cell vaccine against Mycobacterium avium subsp. paratuberculosis in 3 herds of dairy cattle with natural exposure to the organism. J. Am. Vet. Med. Assoc. 242, 663-669. doi: 10.2460/javma.242.5.663

Koets, A., Hoek, A., Langelaar, M., Overdijk, M., Santema, W., Franken, P., et al. (2006). Mycobacterial $70 \mathrm{kD}$ heat-shock protein is an effective subunit vaccine against bovine paratuberculosis. Vaccine 24, 2550-2559. doi: 10.1016/j.vaccine.2005.12.019

Lamont, E. A., Talaat, A. M., Coussens, P. M., Bannantine, J. P., Grohn, Y. T., Katani, R., et al. (2014). Screening of Mycobacterium avium subsp. paratuberculosis mutants for attenuation in a bovine monocyte-derived macrophage model. Front. Cell. Infect. Microbiol. 4:87. doi: 10.3389/fcimb.2014.00087

Li, L., Bannantine, J. P., Zhang, Q., Amonsin, A., May, B. J., Alt, D., et al. (2005). The complete genome sequence of Mycobacterium avium subspecies paratuberculosis. Proc. Natl. Acad. Sci. U.S.A. 102, 12344-12349. doi: 10.1073/pnas.0505662102

Lombard, J. E., Gardner, I. A., Jafarzadeh, S. R., Fossler, C. P., Harris, B., Capsel, R. T., et al. (2013). Herd-level prevalence of Mycobacterium avium subsp. paratuberculosis infection in United States dairy herds in 2007. Prev. Vet. Med. 108, 234-238. doi: 10.1016/j.prevetmed.2012.08.006

Lu, Z., Mitchell, R. M., Smith, R. L., Van Kessel, J. S., Chapagain, P. P., Schukken, Y. H., et al. (2008). The importance of culling in Johne's disease control. J. Theor. Biol. 254, 135-146. doi: 10.1016/j.jtbi.2008.05.008

Lu, Z., Schukken, Y. H., Smith, R. L., and Gröhn, Y. T. (2013). Using vaccination to prevent the invasion of Mycobacterium avium subsp. paratuberculosis in dairy herds: a stochastic simulation study. Prev. Vet. Med. 110, 335-345. doi: 10.1016/ j.prevetmed.2013.01.006

Magombedze, G., Eda, S., and Ganusov, V. V. (2014). Competition for antigen between Th1 and Th2 responses determines the timing of the immune response switch during Mycobaterium avium subspecies paratuberulosis infection in ruminants. PLoS Comput. Biol. 10:e1003414. doi: 10.1371/journal.pcbi. 1003414

Mangtani, P., Abubakar, I., Ariti, C., Beynon, R., Pimpin, L., Fine, P. E., et al. (2014). Protection by BCG vaccine against tuberculosis: a systematic review of randomized controlled trials. Clin. Infect. Dis. 58, 470-480. doi: 10.1093/cid/cit790

Merkal, R. S., and Curran, B. J. (1974). Growth and metabolic characteristics of Mycobacterium paratuberculosis. Appl. Microbiol. 28, 276-279.

Merkal, R. S., Whipple, D. L., Sacks, J. M., and Snyder, G. R. (1987). Prevalence of Mycobacterium paratuberculosis in ileocecal lymph nodes of cattle culled in the United States. J. Am. Vet. Med. Assoc. 190, 676-680.

Muskens, J., Van Zijderveld, F., Eger, A., and Bakker, D. (2002). Evaluation of the long-term immune response in cattle after vaccination against paratuberculosis in two Dutch dairy herds. Vet. Microbiol. 86, 269-278. doi: 10.1016/S03781135(02)00006-8

Park, K. T., Allen, A. J., Bannantine, J. P., Seo, K. S., Hamilton, M. J., Abdellrazeq, G. S., et al. (2011). Evaluation of two mutants of Mycobacterium avium subsp. paratuberculosis as candidates for a live attenuated vaccine for Johne's disease. Vaccine 29, 4709-4719. doi: 10.1016/j.vaccine.2011.04.090

Park, K. T., Allen, A. J., Barrington, G. M., and Davis, W. C. (2014). Deletion of relA abrogates the capacity of Mycobacterium avium paratuberculosis to establish an infection in calves. Front. Cell. Infect. Microbiol. 4:64. doi: 10.3389/fcimb.2014.00064

Park, K. T., Dahl, J. L., Bannantine, J. P., Barletta, R. G., Ahn, J., Allen, A. J., et al. (2008a). Demonstration of allelic exchange in the slow-growing bacterium Mycobacterium avium subsp. paratuberculosis, and generation of mutants with deletions at the $p k n G, r e l A$, and lsr2 loci. Appl. Environ. Microbiol. 74, 1687-1695. doi: 10.1128/AEM.01208-07

Park, S. U., Kathaperumal, K., Mcdonough, S., Akey, B., Huntley, J., Bannantine, J. P., et al. (2008b). Immunization with a DNA vaccine cocktail induces a Th1 response and protects mice against Mycobacterium avium subsp. paratuberculosis challenge. Vaccine 26, 4329-4337. doi: 10.1016/j.vaccine.2008.06.016

Perez De Val, B., Nofrarias, M., Lopez-Soria, S., Garrido, J. M., Vordermeier, H. M., Villarreal-Ramos, B., et al. (2012). Effects of vaccination against paratuberculosis on tuberculosis in goats: diagnostic interferences and cross-protection. BMC Vet. Res. 8:191. doi: 10.1186/1746-6148-8-191

Pradhan, A. K., Mitchell, R. M., Kramer, A. J., Zurakowski, M. J., Fyock, T. L., Whitlock, R. H., et al. (2011). Molecular epidemiology of Mycobacterium avium subsp. paratuberculosis in a longitudinal study of three dairy herds. J. Clin. Microbiol. 49, 893-901. doi: 10.1128/JCM.01107-10

Reddacliff, L., Eppleston, J., Windsor, P., Whittington, R., and Jones, S. (2006). Efficacy of a killed vaccine for the control of paratuberculosis in Australian sheep flocks. Vet. Microbiol. 115, 77-90. doi: 10.1016/j.vetmic.2005.12.021

Saikolappan, S., Estrella, J., Sasindran, S. J., Khan, A., Armitige, L. Y., Jagannath, C., et al. (2012). The $f b p A / s a p M$ double knock out strain of Mycobacterium tuberculosis is highly attenuated and immunogenic in macrophages. PLoS ONE 7:e36198. doi: 10.1371/journal.pone.0036198

Saxegaard, F., and Fodstad, F. H. (1985). Control of paratuberculosis (Johne's disease) in goats by vaccination. Vet. Rec. 116, 439-441. doi: 10.1136/vr.116. 16.439

Scandurra, G. M., De Lisle, G. W., Cavaignac, S. M., Young, M., Kawakami, R. P., and Collins, D. M. (2010). Assessment of live candidate vaccines for paratuberculosis in animal models and macrophages. Infect. Immun. 78, 1383-1389. doi: 10.1128/IAI.01020-09

Scandurra, G. M., Young, M., De Lisle, G. W., and Collins, D. M. (2009). A bovine macrophage screening system for identifying attenuated transposon mutants of Mycobacterium avium subsp. paratuberculosis with vaccine potential. J. Microbiol. Methods 77, 58-62. doi: 10.1016/j.mimet.2009.01.005

Seok, J., Warren, H. S., Cuenca, A. G., Mindrinos, M. N., Baker, H. V., Xu, W., et al. (2013). Genomic responses in mouse models poorly mimic human inflammatory diseases. Proc. Natl. Acad. Sci. U.S.A. 110, 3507-3512. doi: 10.1073/pnas. 1222878110

Seth, M., Lamont, E. A., Janagama, H. K., Widdel, A., Vulchanova, L., Stabel, J. R., et al. (2009). Biomarker discovery in subclinical mycobacterial infections of cattle. PLoS ONE 4:e5478. doi: 10.1371/journal.pone.0005478

Settles, E. W., Kink, J. A., and Talaat, A. (2014). Attenuated strains of Mycobacterium avium subspecies paratuberculosis as vaccine candidates against Johne's disease. Vaccine 32, 2062-2069. doi: 10.1016/j.vaccine.2014.02.010

Shin, S. J., Wu, C. W., Steinberg, H., and Talaat, A. M. (2006). Identification of novel virulence determinants in Mycobacterium paratuberculosis by screening a library of insertional mutants. Infect. Immun. 74, 3825-3833. doi: 10.1128/IAI. 01742-05

Stabel, J. R. (1998). Johne's disease: a hidden threat. J. Dairy Sci. 81, 283-288. doi: 10.3168/jds.S0022-0302(98)75577-8

Stabel, J. R. (2000). Transitions in immune responses to Mycobacterium paratuberculosis. Vet. Microbiol. 77, 465-473. doi: 10.1016/S0378-1135(00)0 0331-X

Stabel, J. R. (2010). "Immunology of paratuberculosis infection and disease," in Paratuberculosis: Organism, Disease, Control, eds M. A. Behr and D. M. Collins (Cambridge: CAB International), 230-243.

Stabel, J. R., Barnhill, A., Bannantine, J. P., Chang, Y. F., and Osman, M. A. (2012). Evaluation of protection in a mouse model after vaccination with Mycobacterium avium subsp. paratuberculois protein cocktails. Vaccine 31, 127-134. doi: 10.1016/j.vaccine.2012.10.090

Stabel, J. R., Waters, W. R., Bannantine, J. P., and Lyashchenko, K. (2011). Mediation of host immune responses after immunization of neonatal calves 
with a heat-killed Mycobacterium avium subsp. paratuberculosis vaccine. Clin. Vaccine Immunol. 18, 2079-2089. doi: 10.1128/CVI.05421-11

Stringer, L. A., Wilson, P. R., Heuer, C., Hunnam, J. C., Verdugo, C., and Mackintosh, C. G. (2013a). Prevalence of Mycobacterium avium subsp. paratuberculosis in farmed red deer (Cervus elaphus) with grossly normal mesenteric lymph nodes. N. Z. Vet. J. 61, 147-152. doi: 10.1080/00480169.2012. 755888

Stringer, L. A., Wilson, P. R., Heuer, C., and Mackintosh, C. G. (2013b). A randomised controlled trial of Silirum vaccine for control of paratuberculosis in farmed red deer. Vet. Rec. 173:551. doi: 10.1136/vr.101799

Sweeney, R. W., Whitlock, R. H., Bowersock, T. L., Cleary, D. L., Meinert, T. R., Habecker, P. L., et al. (2009). Effect of subcutaneous administration of a killed Mycobacterium avium subsp. paratuberculosis vaccine on colonization of tissues following oral exposure to the organism in calves. Am. J. Vet. Res. 70, 493-497. doi: 10.2460/ajvr.70.4.493

Walburger, A., Koul, A., Ferrari, G., Nguyen, L., Prescianotto-Baschong, C., Huygen, K., et al. (2004). Protein kinase G from pathogenic mycobacteria promotes survival within macrophages. Science 304, 1800-1804. doi: 10.1126/science.1099384

Whittington, R. J., Marsh, I. B., Saunders, V., Grant, I. R., Juste, R., Sevilla, I. A., et al. (2011). Culture phenotypes of genomically and geographically diverse Mycobacterium avium subsp. paratuberculosis isolates from different hosts. J. Clin. Microbiol. 49, 1822-1830. doi: 10.1128/JCM. 00210-11

Wu, C. W., Schmoller, S. K., Bannantine, J. P., Eckstein, T. M., Inamine, J. M., Livesey, M., et al. (2009). A novel cell wall lipopeptide is important for biofilm formation and pathogenicity of Mycobacterium avium subspecies paratuberculosis. Microb. Pathog. 46, 222-230. doi: 10.1016/j.micpath.2009. 01.010
Yuan, Y., Lee, R. E., Besra, G. S., Belisle, J. T., and Barry, C. E. 3rd. (1995). Identification of a gene involved in the biosynthesis of cyclopropanated mycolic acids in Mycobacterium tuberculosis. Proc. Natl. Acad. Sci. U.S.A. 92, 6630-6634. doi: 10.1073/pnas.92.14.6630

Zhu, X., Tu, Z. J., Coussens, P. M., Kapur, V., Janagama, H., Naser, S., et al. (2008). Transcriptional analysis of diverse strains Mycobacterium avium subspecies paratuberculosis in primary bovine monocyte derived macrophages. Microbes Infect. 10, 1274-1282. doi: 10.1016/j.micinf.2008.07.025

Conflict of Interest Statement: The authors declare that the research was conducted in the absence of any commercial or financial relationships that could be construed as a potential conflict of interest.

Received: 20 June 2014; accepted: 20 August 2014; published online: 09 September 2014.

Citation: Bannantine JP, Hines ME II, Bermudez LE, Talaat AM, Sreevatsan S, Stabel JR, Chang Y-F, Coussens PM, Barletta RG, Davis WC, Collins DM, Gröhn YT and Kapur $V$ (2014) A rational framework for evaluating the next generation of vaccines against Mycobacterium avium subspecies paratuberculosis. Front. Cell. Infect. Microbiol. 4:126. doi: 10.3389/fcimb.2014.00126

This article was submitted to the journal Frontiers in Cellular and Infection Microbiology.

Copyright (c) 2014 Bannantine, Hines, Bermudez, Talaat, Sreevatsan, Stabel, Chang, Coussens, Barletta, Davis, Collins, Gröhn and Kapur. This is an open-access article distributed under the terms of the Creative Commons Attribution License (CC BY). The use, distribution or reproduction in other forums is permitted, provided the original author(s) or licensor are credited and that the original publication in this journal is cited, in accordance with accepted academic practice. No use, distribution or reproduction is permitted which does not comply with these terms. 